\title{
Prevalence, Morbidity and Mortality of Acute Alcoholic Pancreatitis in the General Hospital of Southern Mexico: Analysis of Five Years (January 2012-December 2016)
}

\section{Concha May AJ and Padrón GA*}

Surgery Service of Hospital General Playa del Carmen, México

\begin{abstract}
Background: From the earliest century, some authors including Friedrech had found that alcohol intake was associated with clinical pictures of acute pancreatitis, which has been confirmed to this day.

Material and method: In order to identify the incidence of acute pancreatitis by alcohol, an observational, retrospective and cross-sectional clinical study was performed at the Playa del Carmen Solidaridad Hospital, Quintana Roo, Mexico.
\end{abstract}

Results: During the study period, 100 cases of acute pancreatitis were collected from which 17 cases of alcoholic pancreatitis. Regarding sex, 3 cases were obtained for females and 14 for males. In terms of age the mean was 40 , median 38, mode 34, SD 7.1, minimum 25 and maximum 52 years. Risk factors: 3 cases for Diabetes mellitus, 3 cases for smoking, 7 cases with hypertriglyceridemia, 5 cases with overweight, and 17 cases with positive alcoholism.

Conclusion: In our hospital the percentage it was $17 \%$ during five years with predominance for male gender in middle age population and ranking third in place of the lipid etiology.

Keywords: Alcohol; Acute pancreatitis; Prevalence; Morbidity; Mortality

\section{Introduction}

From the earliest century, some authors including Friedrech had found that alcohol intake was associated with clinical pictures of acute pancreatitis, which has been confirmed to this day. Nowadays it is widely recognized that pancreatic injure due to alcohol consumption ranges from isolated episodes of acute pancreatitis (AP) to chronic manifestations that with time could move to pancreatic cancer (PC). However, there is not a consensus in the epidemiology and it is no clear how different drinks or dose of alcohol affect to the development of pancreatic diseases and finally, how drinking trigger pancreatic injury only in a minority of alcoholics [1].

It is emphasized that the consumption of alcohol for a long time as well as the amount of alcohol affects significantly both the presentation of acute pancreatitis and the presentation of pancreatic cancer, in such a way that its ingestion increases the incidence of pancreatitis from 2.5 To $3.0 \%$ among alcohol users. Likewise, the amount from 80 to $150 \mathrm{ml}$ per day and for 10 to 15 years leads to the increase of these pathologies $[2,3]$.

The latest Atlanta review of 2012 has reclassified acute and early as well as mild, moderate and severe acute pancreatitis in such a way that mild acute pancreatitis is the most common and the most rapid recovery and with fewer sequelae, such as As happened in most of the cases presented by us [4].

\section{Materials and Methods}

In order to identify the incidence of acute pancreatitis by alcohol, an observational, retrospective and cross-sectional clinical study was performed at the Playa del Carmen Solidaridad Hospital, Quintana Roo, Mexico, during 5 years (January 2012-December 2016). Admission criteria: acute inflammatory pancreatitis associated with ingestion of alcohol. The variables observed were: independent variables: diagnosis of pancreatitis, serum amylase, lipase, dependent variables: age, sex, history, severity ratings used, hospitalization days, morbidity and mortality. Descriptive statistics were used for averages, percentages and standard deviation.

\section{Results}

During the study period, 100 cases of acute pancreatitis were collected from which 17 cases of alcoholic pancreatitis were present (Figure 1) $(2012=3,2013=3,2014: 3,2015: 4$, 2016: 4 cases). Regarding sex, 3 cases were obtained for females and 14 for males. In terms of age the mean was 40 , median 38 , mode 34 , SD 7.1, minimum 25 and maximum 52 years. Risk factors: 3 cases for Diabetes mellitus, 3 cases for smoking, 7 cases with hypertriglyceridemia, 5 cases with overweight, and 17 cases with positive alcoholism. Laboratory tests (Table 1). Ultrasound: 4 cases diagnosed with acute pancreatitis, 3 cases with hepatic steatosis, 1 case with acute chronic pancreatitis, 1 case with free fluid in the abdominal cavity, 1 case report normal and in 7 cases the study was not performed. CT scan performed 8 studies with Balthazar classification: 2 type C, 1 type D and 5 type C Of the 17 cases, 4 suffered complications: 3 cases with peripancreatic collections and 1 case of necrohemorrhagic pancreatitis. Cases were classified with APACHE II 5, Bisap 2 and Ranson 1 cases (Table 2). In terms of management, this was surgical in 1 case of hemorrhagic necrosis and 16 cases with medical treatment. ICU only 3 cases required this service. The days of in-hospital stay were: mean 5, median 4, fashion 3, DE 3.7, minimum 1, maximum 11 days). Prevalence of current consumption of

*Corresponding author: Guillermo Padrón Arredondo, Surgery Service of Hospital General Playa del Carmen, Cerrada Corales 138, Residencial Playa del Sol Solidaridad, Playa del Carmen, Quintana Roo, México, Tel: +01-984-876-2267, +01984-206-1691; E-mail: gpadronarredondo@hotmail.com

Received February 13, 2017; Accepted March 01, 2017; Published March 07 2017

Citation: May CAJ, Padrón GA (2017) Prevalence, Morbidity and Mortality of Acute Alcoholic Pancreatitis in the General Hospital of Southern Mexico: Analysis of Five Years (January 2012-December 2016). Hepatol Pancreat Sci 1: 106.

Copyright: ( 2017 May CAJ, et al. This is an open-access article distributed under the terms of the Creative Commons Attribution License, which permits unrestricted use, distribution, and reproduction in any medium, provided the original author and source are credited. 
Citation: May CAJ, Padrón GA (2017) Prevalence, Morbidity and Mortality of Acute Alcoholic Pancreatitis in the General Hospital of Southern Mexico: Analysis of Five Years (January 2012-December 2016). Hepatol Pancreat Sci 1: 106.

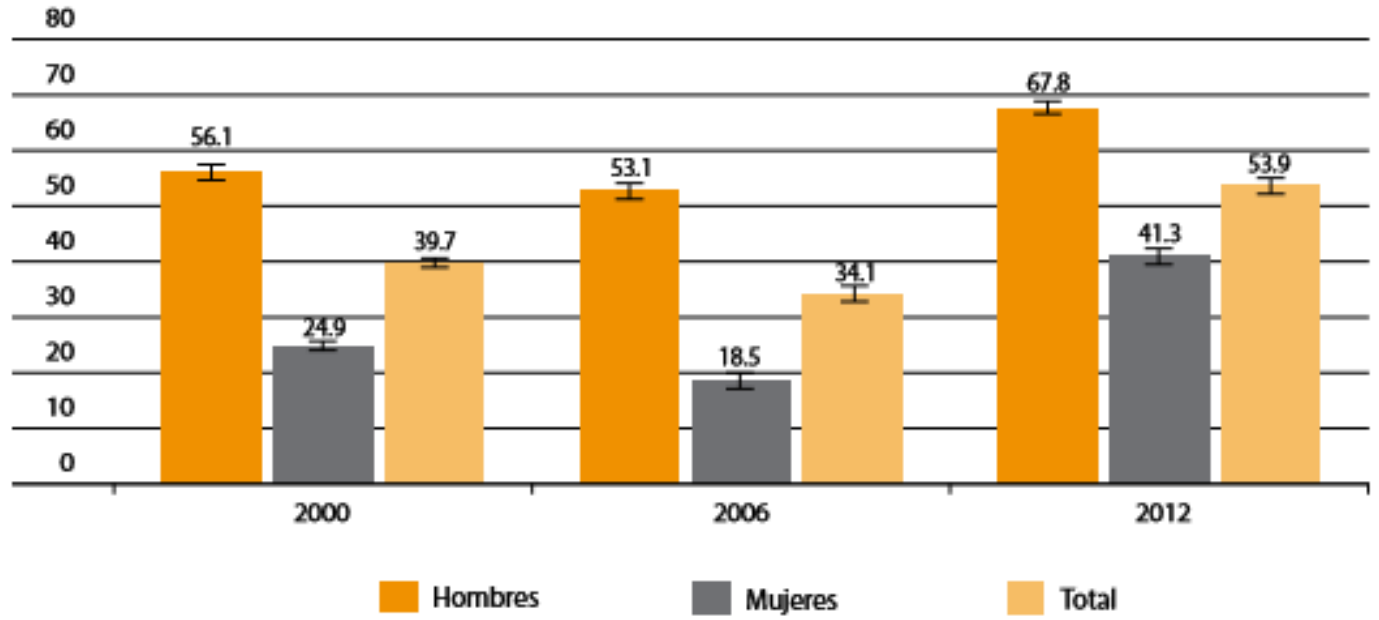

Note: *People who reported alcohol consumption on a daily, weekly, monthly or occasional basis in the last year. 95\% Confidence Intervals

Figure 1: Prevalence of current consumption of alcohol. Population of 20 years or more in Mexico, ENSA 2000, ENSANUT 2006 and 2012.

\begin{tabular}{|c|c|c|c|c|c|c|}
\hline Laboratory Test & Medium & Median & Mode & SD & Minimum & Maximum \\
\hline Hematocrit & 44 & 43 & 52 & 6 & 33 & 53 \\
\hline Leukocytes & 13,038 & 12,900 & $\mathrm{~N} / \mathrm{A}$ & 5 & 5,500 & 22,000 \\
\hline Glucose & 136 & 129 & 112 & 38 & 85 & 118 \\
\hline Total cholesterol & 106 & 85 & $\mathrm{~N} / \mathrm{A}$ & 118 & 85 & 384 \\
\hline Triglicerides & 346 & 84 & $\mathrm{~N} / \mathrm{A}$ & 483 & 67 & 1,324 \\
\hline Lipase & 449 & 293 & $\mathrm{~N} / \mathrm{A}$ & 605 & 108 & 2,120 \\
\hline Amilase & 997 & 537 & $\mathrm{~N} / \mathrm{A}$ & 981 & 105 & 3,378 \\
\hline AST & 56 & 37 & 42 & 68 & 16 & 310 \\
\hline ALT & 51 & 31 & 31 & 51 & 8 & 225 \\
\hline LDH & 218 & $\mathrm{~N} / \mathrm{A}$ & $\mathrm{N} / \mathrm{A}$ & 361 & 183 & 1,042 \\
\hline ALP & 112 & 94 & $\mathrm{~N} / \mathrm{A}$ & 68 & 94 & 271 \\
\hline GGT & 111 & 44 & $\mathrm{~N} / \mathrm{A}$ & 157 & 16 & 523 \\
\hline Calcium & 5 & 8 & $\mathrm{~N} / \mathrm{A}$ & 4.7 & 8 & 10 \\
\hline BUN & 14 & 13 & 13 & 8 & 6 & 37 \\
\hline Creatinine & 1 & 0.88 & 1.24 & 0.94 & 0.52 & 4.6 \\
\hline Albumin & 3.3 & 4 & $\mathrm{~N} / \mathrm{A}$ & 1.8 & 2.2 & 5.2 \\
\hline $\mathrm{DB}$ & 0.69 & 0.45 & 0.45 & 0.87 & 0.09 & 3.77 \\
\hline IB & 1.9 & 0.56 & 0.77 & 5.7 & 0.24 & 23.7 \\
\hline PT & 8.3 & 11.3 & $\mathrm{~N} / \mathrm{A}$ & 7.8 & 0.28 & 19.9 \\
\hline PTT & 16.3 & 20.7 & $\mathrm{~N} / \mathrm{A}$ & 14.9 & 0.56 & 37.6 \\
\hline
\end{tabular}

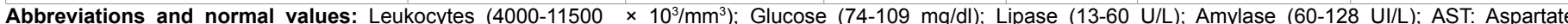
aminotransferase (0-32 U/I); ALT: Alanine aminotransferase (0 -33 U/I); LDH: Lactic dehydrogenase (103-227 U/l); CRP: C-reactive protein (+ or -); ALP: Alkaline phosphatase (64-306 U/I); GGT: Gama glutamil-transpepidase (0-51 U/l ); Calcium (8.5-10.5 mg/dl); BUN: Blood urea nitrogen (10-20 mg/dl); Creatinine (0.4-1.4 mg/ dl); Albumin (3.5-5.3 g/dl); DB: Direct bilirrubin (0-0.20 mg/dl ); IB: Indirect bilirrubin (0.075 mg/dl); PT: Prothrombin time (12.8-15.1 sec); PTT: partial thromboplastin time (24.3-35 sec)

Table 1: Statistical analysis of laboratory analyzes in 17 cases of alcoholic pancreatitis acute of 100 cases acute pancreatitis during five years (2012-2016).

alcohol. Population of 20 years or more in Mexico is shown in (Figure 1). Abuse in alcohol consumption Population aged 20 and over in Mexico (Figure 2).

\section{Discussion}

Incidence increased significantly from 27.6 per 100000 in 1999 to 36.4 in 2010 (average annual increase $=2.7 \%$ per year), there was little trend in mortality ( $0.2 \%$ average annual reduction).
To date, mechanisms that trigger the clinical picture of acute pancreatitis are poorly understood, so that the responsible enzymatic processes continue to be studied as well as the underlying inflammatory response, since the causative etiology of acute pancreatitis is multifactorial [5].

The relationship was linear for $\mathrm{CP}$ and AP in men, but non-linear for AP in women. There was strong evidence supporting a threshold effect for AP in women at the level of alcohol consumption of up to 40 


\begin{tabular}{|c|c|c|c|c|}
\hline \multicolumn{1}{|c|}{ Ultrasound Diagnostic } & \multicolumn{2}{|c|}{ TAC Balthazar } & Miscellaneous Aspects \\
\hline Diagnostic & Cases & Type & Cases & Morbility \\
\hline Acute pancreatitis & 4 & A & 0 & Cases \\
\hline Hepatic steatosis & 3 & B & 0 & Collections peripancreatic \\
\hline Peripancreatic fluid & 2 & C & 2 & Hemorragic necrosis \\
\hline Abdominal free fluid & 1 & D & 1 & Intensive care unit \\
\hline Chronic pancreatitis & 1 & E & 5 & Hospital-stay (days average) \\
\hline Normal study & 1 & Total & $\mathbf{8}$ & Medical management \\
\hline Without US & 5 & & & Surgery treatment \\
\hline Total & $\mathbf{1 7}$ & & & Mortality \\
\hline
\end{tabular}

Table 2: Other results in 17 cases of alcoholic acute pancreatitis out of 100 cases acute pancreatitis during five years (2012-2016).

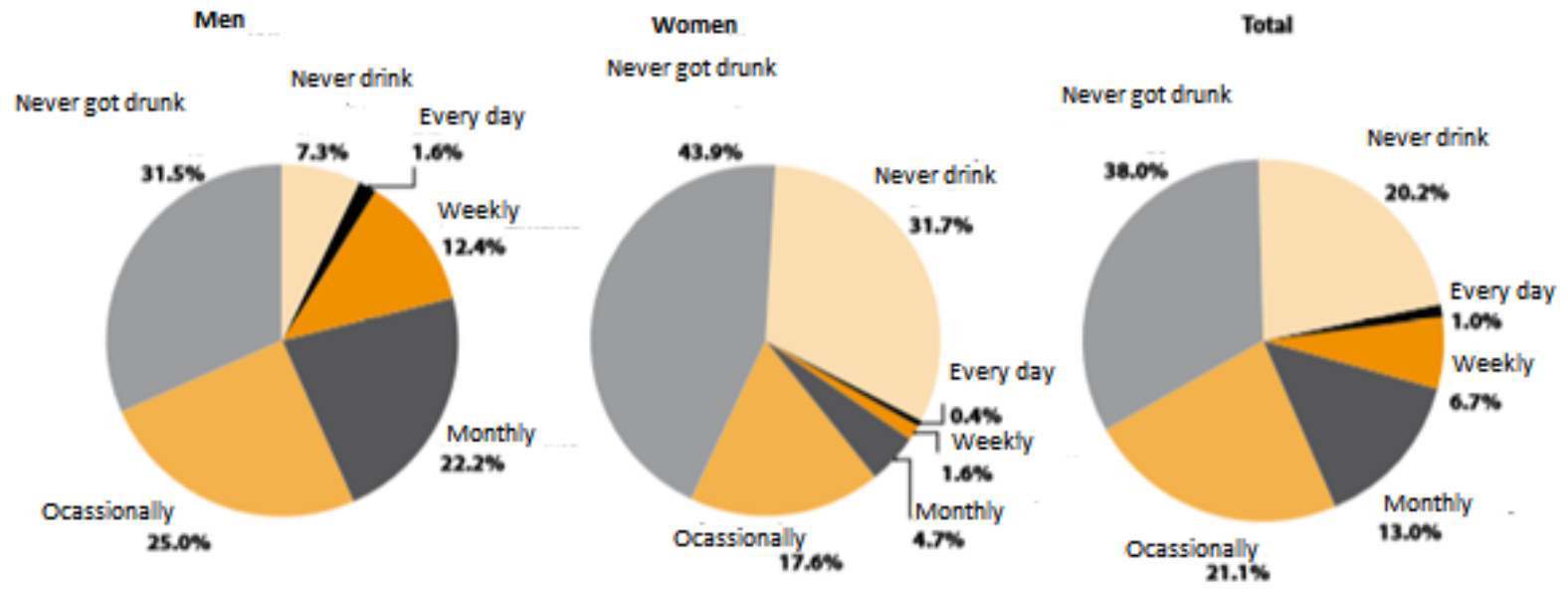

Note: *Consumption of five drinks or more (men) or four drinks or more (women) on a single occasion

Figure 2: Abuse in alcohol consumption. Population aged 20 and over. Mexico, ENSANUT 2012.

$\mathrm{g} /$ day. Beyond $40 \mathrm{~g}$ of pure alcohol/day, the risk of pancreatitis, both acute and chronic, regardless of sex, was higher than previously thought [6].

It is known that alcohol alone does not cause pancreatitis and this is proven by knowing the large number of consumers of alchol and those who do not develop pancreatitis, so that hereditary factor and environmental factors play an interesting role in this alcoholPancreatitis association $[7,8]$.

Because ethanol does not cause pancreatitis directly but only sensitizes the pancreas to cause pancreatitis, so it is assumed that the pancreas has developed self-protective effects against ethanol and some of these protective mechanisms have already been identified $[9,10]$.

In other stuy they found that the appearance of pancreatic pseudocysts was more common in alcohol pancreatitis than in pancreatitis of biliary origin, as well as that endoscopic sphincterotomy could differentiate both entities. In our study, there was only one case of pancreatic pseudocyst and the clinical picture helped us to differentiate both etiologies [11].

In our study, we only found one case of chronic pancreatitis that developed acute pancreatitis, all other cases were first time, however, it is said that alcoholic individuals with chronic pancreatitis tend to develop acute pancreatitis, a situation not observed in our Patients.
Similarly, genetic modification technology in these patients is not available to us for now [12].

The presentation of the pancreatitis cases by alcohol has been increased in the festivities of end of year in our environment due to the high consumption of alcoholic drinks and in individuals who ingestion of these drinks of constant form. Other authors have found the relation of festive periods and the association with morbimortality by pancreatitis $[13,14]$.

Acute pancreatitis in an alcoholic is generally secondary to chronic pancreatitis. Determinants of individual susceptibility to alcoholic pancreatitis still remain uncertain. The studies in progress lead us to a clear understanding of the mechanisms involved in the pathogenesis of alcoholic pancreatitis. In the case of intractable pain, an intrathecal narcotics pump may be offered. In those patients in whom medical therapy failed to obtain persistent pain relief, a surgical approach should be preferred over an endoscopic approach [15].

\section{Conclusion}

In our hospital the percentage it was $17 \%$ during five years with predominance for male gender in middle age population and ranking third in place of the lipid etiology.

\section{Conflict of Interest}


Citation: May CAJ, Padrón GA (2017) Prevalence, Morbidity and Mortality of Acute Alcoholic Pancreatitis in the General Hospital of Southern Mexico: Analysis of Five Years (January 2012-December 2016). Hepatol Pancreat Sci 1: 106.

The authors declare no conflict of interest.

\section{Financing}

No financing has been received of any kind

\section{References}

1. Friedreich $N(1878)$ Disease of the pancreas. Cyclopoedia of the practice of medicine. William Wood, New York.

2. Herreros-Villanueva M, Hijona E, Bañales JM, Cosme A, Bujanda L (2013) Alcohol consumption on pancreatic diseases. World J Gastroenterol 19: 638-647.

3. Vonlaufen A, Spahr L, Apte MV, Frossard JL (2014) Alcoholic pancreatitis: A tale of spirits and bacteria. World J Gastrointest Pathophysiol 5: 82-90.

4. Acute Pancreatitis Classification Working Group (2013) Classification of acute pancreatitis-2012: Revision of the Atlanta classification and definitions by international consensus. Gut 62: 102-111.

5. Pandol SJ, Gorelick FS, Gerloff A, Lugea A (2010) Alcohol abuse, endoplasmic reticulum stress and pancreatitis. Dig Dis 28: 776-782.

6. Roberts SE, Akbari A, Thorne K, Atkinson M, Evans PA (2013) The incidence of acute pancreatitis: impact of social deprivation, alcohol consumption, seasonal and demographic factors. Aliment Pharmacol Ther 38: 539-548.

7. Samokhvalov AV, Rehm J, Roerecke M (2015) Alcohol consumption as a risk factor for acute and chronic pancreatitis: A systematic review and a series of meta-analyses. EBioMedicine 2: 1996-2002.

8. Pandol SJ, Lugea A, Mareninova OA, Smoot D, Gorelick FS, et al. (2011) Investigating the pathobiology of alcoholic pancreatitis. Alcohol Clin Exp Res
35: $830-837$.

9. Clemens DL, Schneider KJ, Arkfeld ChK, Grode JR, Wells MA, et al. (2016) Alcoholic pancreatitis: New insights into the pathogenesis and treatment. World J Gastrointest Pathophysiol 7: 48-58.

10. Clemens DL, Wells MA, Schneider KJ, Singh S (2014) Molecular mechanisms of alcohol associated pancreatitis. World J Gastrointest Pathophysiol 5: 14757.

11. Cho JH, Kim TN, Kim SB (2015) Comparison of clinical course and outcome of acute pancreatitis according to the two main etiologies: Alcohol and gallstone. BMC Gastroenterology 15: 87.

12. Gu H, Werner J, Bergmann F, Whitcomb DC, Büchler MW, et al. (2013) Necroinflammatory response of pancreatic acinar cells in the pathogenesis of acute alcoholic pancreatitis. Cell Death Dis 4: e816.

13. Roberts SE, Thorne K, Evans PA, Akbar A, Samuel DG, et al. (2014) Mortality following acute pancreatitis: social deprivation, hospital size and time of admission: record linkage study. BMC Gastroenterology 14: 153.

14. Andersen AM, Novovic S, Ersbøll AK, Hansen MB (2008) Mortality in alcohol and biliary acute pancreatitis. Pancreas 36: 432-434.

15. Pezzilli R, Morselli-Labate AM (2009) Alcoholic pancreatitis: Pathogenesis incidence and treatment with special reference to the associated pain. Int $J$ Environ Res Public Health 6: 2763-2782. 